\title{
Overexpressed genes associated with hormones in terminal ductal lobular units identified by global transcriptome analysis: An insight into the anatomic origin of breast cancer
}

\author{
JIANMIN YANG ${ }^{1}$, HAIJING YU ${ }^{1}$, LIANG ZHANG ${ }^{2}$, HUA DENG $^{2}$, QI WANG ${ }^{1}$, \\ WENPING LI ${ }^{1}$, ANQIN ZHANG ${ }^{1}$, HONGYI GAO ${ }^{3}$ and AIHUA YIN ${ }^{4}$ \\ ${ }^{1}$ Breast Disease Center, ${ }^{2}$ Translational Medicine Center, ${ }^{3}$ Department of Pathology and ${ }^{4}$ Medical Genetics Center, \\ Guangdong Women and Children's Hospital, Guangzhou, Guangdong 511400, P.R. China
}

Received October 4, 2015; Accepted November 10, 2015

DOI: $10.3892 /$ or.2015.4523

\begin{abstract}
Although human breast ducts and terminal ductal lobular units (TDLUs) share the same cell types, ample evidence shows that TDLUs are the predominant site for the origin of breast cancer. Yet, there is still limited information concerning the molecular mechanisms. Analysis of transcriptomic profiles in TDLUs may provide insight into early breast tumorigenesis. We compared genome-wide expression profiles of 8 matched sets of breast main duct and TDLU samples, using significance analysis of microarray (SAM) software to screen differentially expressed genes (DEGs) with fold-change $>2.0$ and q-value $<0.05$. Moreover, we used Gene Ontology for functional enrichment analysis. We identified 472 DEGs between the two tissue types, and confirmed 17 randomly chosen DEGs by quantitative reverse transcription-PCR (qRT-PCR). Notably, hormone-related pathways were highly enriched in the TDLU samples, including various hormone-related DEGs that are associated with breast carcinogenesis and tumor progression. Oncogenic upregulation in TDLUs indicates a potential inappropriate or excessive response to successive hormone stimulus during the proliferation, differentiation and lactation cycles of the human mammary gland. Imbalanced hormone reactions may finally result in the early onset of neoplastic transformation that occurs mostly in breast TDLUs.
\end{abstract}

Correspondence to: Professor Qi Wang, Breast Disease Center, Guangdong Women and Children's Hospital, 521 Xingnan Road, Guangzhou, Guangdong 511400, P.R. China

E-mail: wangqigz@hotmail.com

Abbreviations: TDLU, terminal ductal lobular unit; DEGs, differentially expressed genes; qRT-PCR, quantitative reverse transcription-polymerase chain reaction

Key words: terminal ductal lobular unit, breast cancer, hormones, differentially expressed genes, global transcriptome profiling

\section{Introduction}

Breast cancer is the most common malignancy and the leading cause of cancer-related deaths among women worldwide. Morphologically, breast cancer can be classified as ductal carcinoma or lobular carcinoma. Although ductal carcinomas were initially thought to arise from the ductal system, current evidence shows that most benign and malignant breast lesions originate from the same anatomical compartment, the terminal ductal lobular units (TDLUs). Hyperplasic or atypical hyperplasic epithelia that line TDLUs are believed to be carcinoma precursor or premalignant cells. Histologically, a TDLU has both ductal and lobular components; premalignant TDLU cells can further develop to ductal or lobular carcinoma (1-4). The lobules are the milk-producing breast structures, of which the terminal lobules are the TDLUs (5). Notably, TDLUs are predominant site of origin of breast cancer even though the ducts and TDLUs consist of the same general cell types.

Gene expression profiling can provide valuable information concerning carcinogenesis, classification, diagnosis and treatment of tumors, and has been widely used to investigate the molecular mechanisms of various cancer types, particularly for breast cancer in Western countries. However, most microarray studies of breast cancers have mainly focused on cancer itself (6-8). Reports of microarray analyses of TDLUs and breast cancer etiology are scarce. For example, Tripathi et al compared gene expression profiles between histologically normal TDLU epithelium of breast cancer patients and cancer-free controls. They found that global gene expression abnormalities already existed in the non-cancerous epithelium of breast cancer subjects and in early stage carcinomas (9).

The present study aimed to survey genes that are differentially regulated in breast TDLU tissues and main duct controls, using genome-wide expression profiling. A total of 472 differentially expressed genes (DEGs) were identified between the two groups. Notably, hormone metabolism and response pathways were highly enriched in the TDLU samples, including various hormone-related DEGs that are reportedly associated with malignant transformation and progression in breast cancers. The marked differences in transcriptomic patterns between the human breast duct and TDLU samples 
Table I. Primers for qRT-PCR validation of differentially expressed genes.

\begin{tabular}{lll}
\hline Gene & GeneBank no. & \\
\hline ADIPOQ & NM_004797.3 & F: GCCTTCCGCAGTGTAGGCTT, R: AGCCAGATGGTGTGGCTTGG \\
ADORA2B & NM_000676.2 & F: GCTACACCTCACAAGGAAAT, R: ATCCTTGGAGCCTACTACTG \\
BMP7 & NM_001719.2 & F: ACCCGTGGCTGTCTCTTCA, R: AGGCAAGGCAGGCTTACAC \\
$C A 4$ & NM_000717.3 & F: GAAATTGCGGTGCTGGCCT, R: AGCGGAAGTAGTGCCTCAGT \\
$C C L 19$ & NM_006274.2 & F: GGGTGCCTGCTGTAGTGTTC, R: CTCGGTTCCCCAGGTTAGGT \\
$C C L 21$ & NM_002989.2 & F: GCCTTGCCACACTCTTTCTC, R: TCCTGCTGCCTCCTCTCAT \\
$C E L$ & NM_001807.3 & F: TGCCAAGAGTGCCAAGACC, R: TCTCCAGGTAGCCGCTGTT \\
ECSCR & NM_001077693.2 & F: AGAGACGGAGGCACAAGCA, R: AGCACAGTCTCTGACGTGGG \\
FABP4 & NM_001442.2 & F: GTCACAGCACCCTCCTGAA, R: CCATGCCAGCCACTTTCCT \\
HSD11B2 & NM_000196.3 & F: CTGGCTGCTTCAAGACAGAGT, R: AGGCAGGTAGTAGTGGATGAA \\
ITGB3 & NM_000212.2 & F: GTGACCTGAAGGAGAATCTGC, R: TTCTTCGAATCATCTGGCC \\
LAMA1 & NM_005559.3 & F: TGGCTATCCTGCTGGTGTGA, R: CGAACGCTCTGCTGAAGTCA \\
LILRB3 & NM_006864.2 & F: CAGAGCCCACACGATGAAG, R: AGTCTCCTTCTGCTGAGTGT \\
LOX & NM_001178102.1 & F: ATCCCTGAAATGTCTGCCT, R: CCAGGAGGGGGATGAATGT \\
S100A8 & NM_002964.4 & F: TGAAGAAATTGCTAGAGAC, R: CTTTATCACCAGAATGAGGA \\
S100A9 & NM_002965.3 & F: ATGCTGATGGCGAGGCTAAC, R: ACTGTGGTCTTAGGGGGTGC \\
WNT5A & NM_003392.3 & F: CGTTAGCAGCATCAGTCCA, R: CTGTGCCTTCGTGCCTATT \\
\hline
\end{tabular}

F, forward; R, reverse.

are likely to reflect differences in responses to microenvironment alteration. This study provides important clues to the hormone-driven carcinogenic process that starts in the TDLU, and may facilitate the development of novel targeted therapeutics for breast cancer.

\section{Materials and methods}

Clinical specimens. This study was approved by the Medical Ethics Committees of the Guangdong Women and Children's Hospital. Written informed consent was obtained from all participants. We collected 8 matched pairs of breast main duct and TDLU tissue samples from 8 premenopausal patients with benign papilloma who underwent surgical treatment. Pathological examination was performed to confirm histological normalcy of the uninvolved tissues. The main ducts were extracted along with the blue blot after injection of $0.2 \mathrm{ml}$ of methylenum caeruleum dye into the discharge hole during resection. The TDLU samples were collected by incising $2 \times 2 \mathrm{~mm}$ sections of the non-blue-stained glandular tissue at the terminal of specimens. Immediately after collection, all samples were rapidly preserved in RNAlater RNA stabilization reagent (Qiagen, Inc., Chatsworth, CA, USA); a portion of each sample was used for histological analysis.

Global gene expression profiling. Briefly, total RNA was extracted using TRIzol reagent (Invitrogen, Gaithersburg, MD, USA) according to the manufacturer's instructions. RNA quality was assessed by formaldehyde agarose gel electrophoresis; RNA concentration was measured using spectrophotometry (Nanodrop, Wilmington, DE, USA). We then amplified and labelled $100 \mathrm{ng}$ of total RNA of each sample using Agilent Low Input Quick Amp Labeling kit.
Fluorescence (Cy5 or $\mathrm{Cy} 3$ ) labeled cRNA was hybridized to Agilent human $8 \times 60 \mathrm{~K}$ microarrays at $65^{\circ} \mathrm{C}$ for $16 \mathrm{~h}$ in a hybridization oven. Hybridized slides were washed and scanned using an Agilent microarray scanner. Raw data of the obtained images were extracted using Agilent Feature Extraction software and analyzed using Agilent GeneSpring GX 11.5 software. The data were deposited in the NCBI Gene Expression Omnibus (http://www.ncbi.nlm.nih.gov/geo) under accession number GSE39021.

Quantitative reverse transcription-PCR ( $q R T-P C R)$ validation. Briefly, up to $1 \mu \mathrm{g}$ of DNase I-treated total RNAs per sample was reverse-transcribed into cDNA using oligo $(\mathrm{dT})_{12}$ and M-MLV reverse transcriptase (Life Technologies) following standard procedures. The derived cDNA was further amplified in triplicates using a LightCycler ${ }^{\circledR} 480$ Real-Time PCR system (Roche Applied Science). The 20- $\mu 1$ reaction mixture contained 1X PCR buffer $\left(\mathrm{Mg}^{2+}\right.$ Plus), $200 \mu \mathrm{M}$ of each $\mathrm{dNTP}, 0.5 \mu \mathrm{M}$ of the forward primer, $0.5 \mu \mathrm{M}$ of the reverse primer (Table I), and Eva Green Master Mix. PCR conditions consisted of $95^{\circ} \mathrm{C}$ for $4 \mathrm{~min} ; 45$ cycles of $94^{\circ} \mathrm{C}$ for $30 \mathrm{sec}, 58^{\circ} \mathrm{C}$ for $30 \mathrm{sec}$ and $72^{\circ} \mathrm{C}$ for $30 \mathrm{sec}$; followed by $1 \mathrm{cycle}$ of $72^{\circ} \mathrm{C}$ for $8 \mathrm{~min}$. Relative quantification of expression was analyzed by the $2^{-\Delta \Delta \mathrm{CT}}$ method.

Statistical analysis. Genes were regarded as expressed and retained for further analysis if the probe intensities were $>400$ after global median normalization. DEGs were screened using two class unpaired analysis in the SAM software. Thresholds were set at a fold-change $>2.0$ and q-value $<0.05$. Significant enrichment of Gene Ontology (GO) terms was analyzed using hyper-geometric distribution in the $\mathrm{R}$ language software package. 


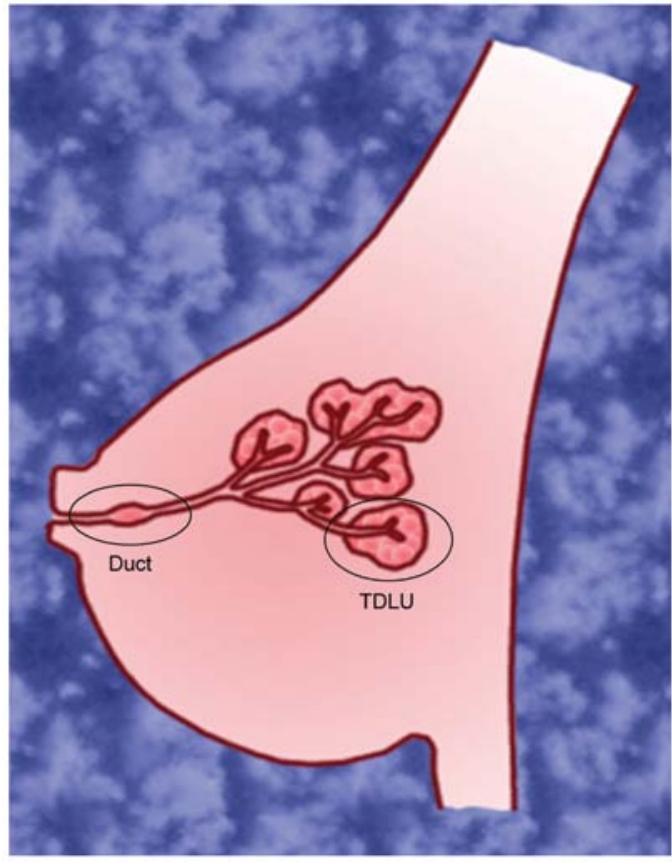

Figure 1. Anatomic sites of the human normal main duct and TDLUs. Clinical samples from these two compartments were collected to compare their gene expression profiles. TDLUs, terminal ductal lobular units.

\section{Results}

Differential transcriptomic patterns between breast main duct and TDLU tissues. The anatomic sites of the breast main duct and TDLUs are shown in Fig. 1. Total extracted RNA from clinical samples was used for microarray analysis. A total of 472 mRNA transcripts were identified as being significantly differentially expressed. Supervised hierarchical clustering of the 472 DEGs showed distinct patterns between the breast duct and TDLU samples (Fig. 2). Among them, 230 genes were highly expressed in the TDLU tissues, and 242 genes were highly expressed in the main duct tissues. The significant enrichment analysis of GO terms for the DEGs using the $\mathrm{R}$ language package software showed that these 472 hits were involved in distinct biological processes. Notably, the top 6 pathways, hormone metabolic process, regulation of hormone levels, response to hormone stimulus, cellular hormone metabolic process, response to endogenous stimulus and steroid metabolic process, are implicated in hormone metabolism and response. As these genes are related and greatly overlap, we combined them into a 'hormone-related' pathway with a total of 18 genes (Table II).

Correlation between microarray analysis and $q R T-P C R$ results. To confirm the microarray results, 17 DEGs identified by microarray were randomly chosen for validation using qRT-PCR. These genes included $A D I P O Q, A D O R A 2 B$, BMP7, CA4, CCL19, ITGB3, CCL21, CEL, ECSCR, FABP4, LOX, S100A8, HSD11B2, LAMA1, LILRB3, S100A9 and $W N T 5 A$. The unchanged expression of dehydrogenase $(G A P D H)$ between the two tissues was used as a reference gene. The fold-change of expression for each gene between the breast main duct and TDLU tissues was measured using the same batch of samples as in the microarray. Ratios measured

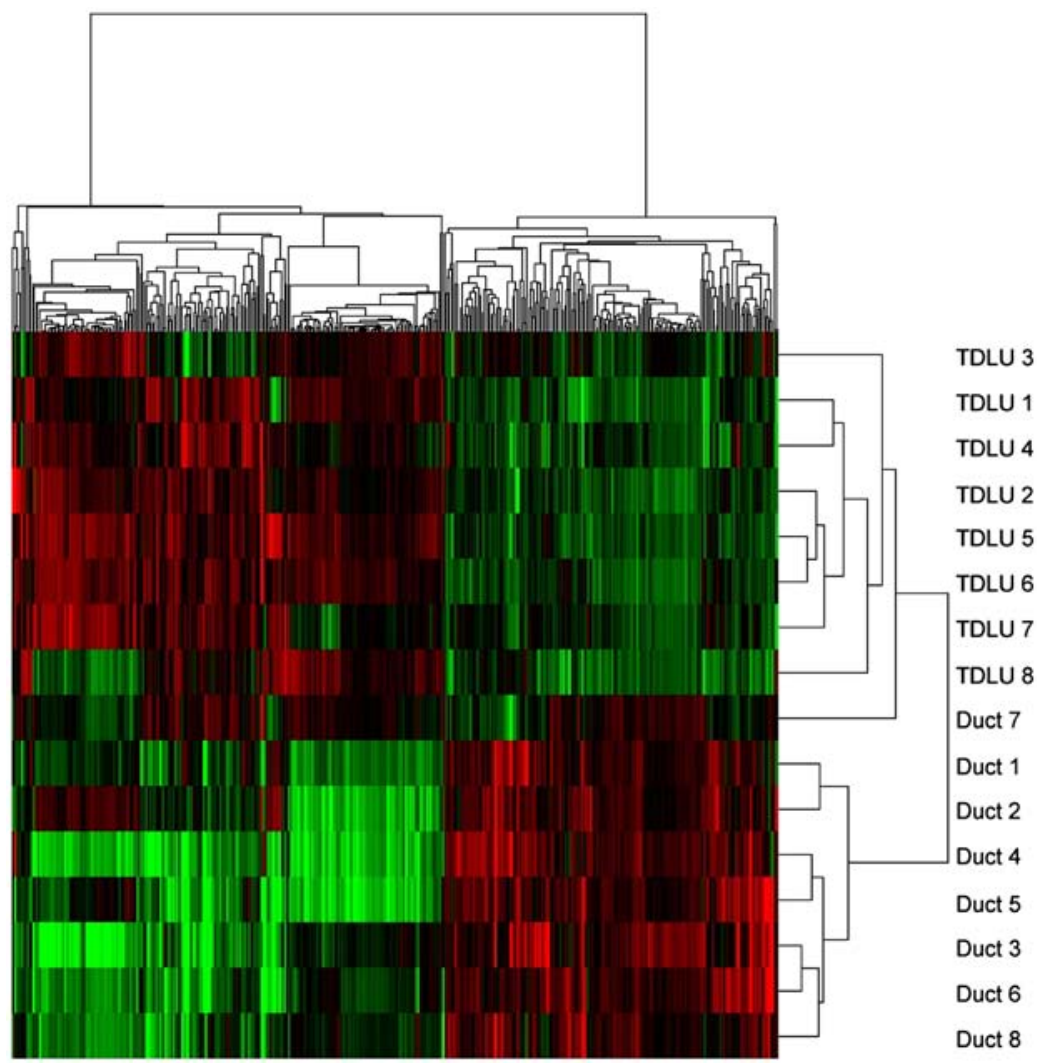

Figure 2. Cluster analysis of genes with significantly different expression in the 8 matched pairs of breast duct and TDLU tissues. Green, downregulated genes; red, upregulated gene. TDLU, terminal ductal lobular unit. 
Table II. List of hormone-related genes overexpressed in the TDLU samples enriched by GO term analysis.

\begin{tabular}{lccl}
\hline Gene & q-value $(\%)$ & Fold-change $^{\mathrm{a}}$ & \\
\hline CA4 & 0 & 3.66 & Description \\
CYP26A1 & 0 & 3.36 & Carbonic anhydrase IV \\
FABP4 & 0.54 & 2.87 & Cytochrome P450, family 26, subfamily A, polypeptide 1 \\
CRYM & 1.01 & 2.61 & Fatty acid binding protein 4, adipocyte \\
FAM3B & 1.51 & 2.52 & Crystallin, mu \\
ADCY1 & 1.51 & 2.01 & Family with sequence similarity 3, member B \\
EGR2 & 1.83 & 2.04 & Adenylate cyclase 1 (brain) \\
HSDIIB2 & 2.61 & 2.16 & Early growth response 2 \\
ADIPOQ & 3.44 & 2.52 & Hydroxysteroid (11- $\beta$ ) dehydrogenase 2 \\
SCG5 & 4.11 & 2.66 & Adiponectin, C1Q and collagen domain containing \\
IGF2 & 5.14 & 2.39 & Insulin-like growth factor 2 \\
HMGCS2 & 6.82 & 4.79 & 3-Hydroxy-3-methylglutaryl-CoA synthase 2 (mitochondrial) \\
SERPINA1 & 7.52 & 2.56 & Serpin peptidase inhibitor, clade A, member 1 \\
CA2 & 8.38 & 2.43 & Carbonic anhydrase II \\
LEP & 8.38 & 2.28 & Leptin \\
SERPINA6 & 9.97 & 3.86 & Serpin peptidase inhibitor, clade A, member 6 \\
UGT $2 B 11$ & 11.20 & 4.27 & UDP glucuronosyltransferase 2 family, polypeptide B11 \\
DHRS2 & 11.49 & 4.02 & Dehydrogenase/reductase (SDR family) member 2 \\
& & &
\end{tabular}

${ }^{\text {a}}$ Fold-change indicates gene expression levels in the terminal ductal lobular unit (TDLU) samples relative to the breast duct samples.

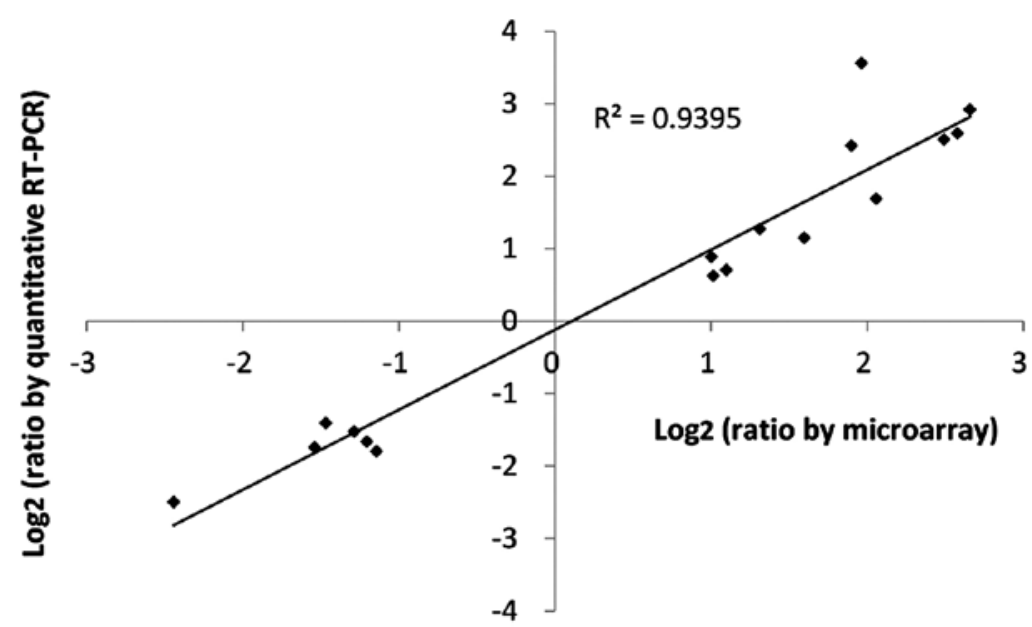

Figure 3. Quantitative RT-PCR validation of 17 differentially expressed genes identified by microarray. Gene expression levels of each gene were measured in triplicate and are shown as average fold-change relative to $G A D P H$ used as the internal control. $\mathrm{R}$ indicates the Pearson's linear correlation coefficient value.

by the microarray were highly consistent with the data from qRT-PCR, which confirmed marked differences in gene expression profiles between the two groups (Fig. 3).

\section{Discussion}

Women undergo multiple cycles of growth and differentiation of mammary glands through their lifetimes. Although the basic structure of the mammary gland is composed of ducts and lobules, their phenotypes and gene expression patterns alter greatly with age and pregnancy. Considering that breast differentiation and development are relatively stable in multiparous women, premenopausal multiparous women were selected to analyze gene expression profiles of breast duct and TDLU tissues. Significantly different transcriptomes were identified between the two structures and confirmed by qRT-PCR, which indicated fundamental differences in physiological functions and biochemical processes.

The breast is a hormone-dependent organ, and breast carcinomas are closely modulated by hormones, not only steroid hormones, but any chemical substances produced in the body with essential biological functions for life such as metabolism, growth, development, differentiation and reproduction. We found that 230 genes were highly expressed in the TDLUs, the 
functional units of the breast. Among the functions associated with these genes, the top 6 were related to hormone metabolism and hormone response. These hormone-related upregulated genes included HMGCS2, UGT2B11, DHRS2, SERPINA6, CA4, CYP26A1, FABP4, SCG5, CRYM, SERPINA1, FAM3B, $A D I P O Q, C A 2, I G F 2, L E P, H S D 11 B 2, E G R 2$ and $A D C Y 1$. Some of these are closely associated with tumorigenesis and cancer development, which suggests an explanation for the higher production of cancer cells in TDLUs.

The protein encoded by $H M G C S 2,3$-hydroxy-3-methylglutaryl-CoA synthase 2 (mitochondrial), belongs to the HMG-CoA synthase family. It functions as a mitochondrial enzyme that catalyzes the first ketogenesis reaction in various hormonal and metabolic situations. Recently, Martinez-Outschoorn et al reported that fibroblasts that overexpress MGCS2 in tumor stroma effectively promote anabolic tumor growth, and its overexpression in MDA-MB-231 breast cancer cells markedly increased tumor development and metastatic capacity by driving increased mitochondrial biogenesis. Thus, the ketogenesis pathway is a potential target in cancer intervention and therapeutics. These results clearly indicate that HMGCS2 is a bona fide metabolic oncogene (10). Here, HMGCS2 was the most highly upregulated gene (4.79-fold) in the TDLU samples, which may drive breast cancer development and progression via the ketone metabolism pathway.

Fatty acid binding protein-4 (FABP4) is encoded and found in adipocytes. Fatty acid binding proteins are a family of highly conserved, cy toplasmic proteins that bind long-chain fatty acids and play key roles in fatty acid uptake, transport and metabolism. The role of lipid metabolism in cancer has recently drawn great attention. FABP4 was found to be upregulated in omental metastases compared with primary ovarian tumors, using a protein array. FABP4 deficiency significantly inhibited tumor metastasis in mice, indicating its important role in ovarian cancer metastasis. This study showed that stromal adipocytes provide fatty acids for rapid cancer cell growth, suggesting that lipid metabolism could be a target in the treatment of cancer (11). Substantially higher levels of serum FABP4 have been identified in breast cancer patients when compared with healthy controls, and FABP4 has been positively associated with tumor size and nodal status. These data demonstrate that high FABP4 levels are significantly associated with breast cancer risk and adverse tumor characteristics (12). Similar correlations have also been determined in prostate cancer (13). These findings are further supported by previous observations that FABP4 expression was the highest in the HER2-positive breast cancer subtype. FABP4 positivity was associated with significantly shorter disease-free survival and overall survival in triple-negative breast cancer (14). Thus, selective inhibition of FABP4 may be a potential anticancer strategy for various breast cancer subtypes. The breast is an endocrine organ, consisting of adipose tissue, which can influence tumor growth or differentiation by adipose tissue-derived hormones.

Glucocorticoids have an anti-proliferative effect in many tumors including breast cancer cells $(15,16)$. Hydroxysteroid (11- $\beta$ ) dehydrogenase-2 (HSD11B2) potently inactivates hormonally active glucocorticoids to comparatively less active metabolites. HSD11B2 has thereby been postulated to protect cells from the growth-inhibiting and or pro-apoptotic effects of glucocorticoids involved in the protein kinase A and protein kinase $\mathrm{C}$ signaling pathways (17). Increased HSD11B2 expression has an obvious function in cells that acquire anchorage-independent growth and acinar-conformational disruption. A reduced HSD11B2 protein level by specific siRNAs resulted in diminished breast cell carcinogenesis (18). Similarly, inhibition of HSD11B2 expression was found to suppress the proliferation of breast cancer PMC42 cells (19). These results were further supported by detection of HSD11B2 overexpression in breast cancer cell lines and breast tumors, leading to an increased cell growth of $50-120 \%(20,21)$. Thus, HSD11B2 upregulation is postulated to have a proliferative role on breast cancer cells through impairment of the antiproliferative activity of glucocorticoids.

Leptin (LEP), an adipocyte-derived cytokine, plays a major role in the regulation of food intake, energy expenditure and body weight through the leptin receptor. It also has endocrine functions, and affects immune responses, hematopoiesis, angiogenesis and wound healing. Although leptin is mainly expressed by white adipocytes, it is also expressed by other tissues including mammary epithelial cells (22). Leptin mRNA expression was significantly higher in breast cancer cells than that in non-cancerous tissues and increased expression of leptin and the leptin receptor were found during oncogenic transformation of benign cells $(23,24)$. In addition, strong leptin protein expression was detected in most breast cancer cells but not in normal epithelium, using immunohistochemical staining (25). Serum leptin levels have also been found to be highly elevated in breast cancer patients compared with levels in healthy controls (26). Furthermore, an in vivo study showed that leptin directly contributed to the development of mammary tumors in a leptin-deficient mouse model (27). These observations strongly suggest that leptin production is enhanced in mammary glands during tumorigenesis. Leptin increases anchorage-dependent breast cell proliferation, and may promote carcinogenesis and progression of breast cancer by activating pathways that include signal transducers and activators of transcription 3 (STAT3), extracellular signalregulated kinase (ERK) and transcript activator protein 1 (AP-1) (28). Functionally blocking leptin signaling may be an effective approach to prevent and treat breast cancer.

The other upregulated genes in TDLU tissues have been shown to affect breast tumorigenesis and progression. Insulinlike growth factor 2 (IGF-2) is a mitogenic peptide hormone; its overexpression occurs in many cancer types and it is associated with poor patient prognosis. IGF-2 is thought to control normal cell growth and development, and stimulates breast cancer proliferation (29-31). Adipocytes have been shown to secrete levels of IGF-2 that are sufficient to promote proliferation of MCF-7 breast cancer cells (32). Approximately $20-30 \%$ of breast cancers show amplification and elevated expression of ErbB2 (i.e., HER-2), which is associated with poor clinical outcomes. Early growth response 2 (EGR2) is a zinc finger transcription factor. An EGR2 binding site is reportedly found within the $E r b B 2$ promoter, and EGR2 is reportedly upregulated during $E r b B 2^{+}$tumor induction (33). Furthermore, overexpression of EGR2 has been reported in ErbB2-expressing breast cancer cell lines. These findings implicate EGR2-ErbB2 signaling in mammary tumor formation (34). 
Large bodies of clinical and epidemiological evidence shows the critical role of hormones in mammary gland development and tumorigenesis $(35,36)$. As women age, their breast lobules and TDLU structures regress. This results in reduced breast cancer risk. Conversely, a gradual increase in breast cancer risk is associated with increasing numbers of lobule units (37). Obviously, enhanced oncogene expression in TDLUs sensitizes the lobular epithelium to the proliferative effects of successive hormone stimulus. Multiple accumulated genetic changes may drive a benign-malignant progression, finally resulting in the onset of breast cancer originating in the TDLU. Our findings should be verified by studies with larger cohorts.

In summary, we identified a cluster of upregulated genes involved in hormone metabolism and hormone stimulus response pathways in TDLU samples by global transcriptome analysis. Notably, the hormone stimulus of these elevated DEGs is potentially oncogenic, and may contribute to the greater incidence of breast carcinogenesis occurring in TDLU tissue.

\section{Acknowledgements}

The present study was supported by a grant from the Guangdong Science and Technology Program, China (2012B031800174).

\section{References}

1. Wellings SR and Jensen HM: On the origin and progression of ductal carcinoma in the human breast. J Natl Cancer Inst 50: $1111-1118,1973$

2. Wellings SR, Jensen HM and Marcum RG: An atlas of subgross pathology of the human breast with special reference to possible precancerous lesions. J Natl Cancer Inst 55: 231-273, 1975.

3. Wellings SR: A hypothesis of the origin of human breast cancer from the terminal ductal lobular unit. Pathol Res Pract 166: 515-535, 1980.

4. Allred DC, Mohsin SK and Fuqua SA: Histological and biological evolution of human premalignant breast disease. Endocr Relat Cancer 8: 47-61, 2001.

5. Musumeci G, Castrogiovanni P, Szychlinska MA, Aiello FC, Vecchio GM, Salvatorelli L, Magro G and Imbesi R: Mammary gland: From embryogenesis to adult life. Acta Histochem 117: 379-385, 2015

6. Ma XJ, Salunga R, Tuggle JT, Gaudet J, Enright E, McQuary P, Payette T, Pistone M, Stecker K, Zhang BM, et al: Gene expression profiles of human breast cancer progression. Proc Natl Acad Sci USA 100: 5974-5979, 2003.

7. Sotiriou C, Neo SY, McShane LM, Korn EL, Long PM, Jazaeri A, Martiat P, Fox SB, Harris AL and Liu ET: Breast cancer classification and prognosis based on gene expression profiles from a population-based study. Proc Natl Acad Sci USA 100: 10393-10398, 2003.

8. Fu J, Allen W, Xia A, Ma Z and Qi X: Identification of biomarkers in breast cancer by gene expression profiling using human tissues. Genom Data 2: 299-301, 2014.

9. Tripathi A, King C, de la Morenas A, Perry VK, Burke B, Antoine GA, Hirsch EF, Kavanah M, Mendez J, Stone M, et al: Gene expression abnormalities in histologically normal breast epithelium of breast cancer patients. Int J Cancer 122: 1557-1566, 2008.

10. Martinez-Outschoorn UE, Lin Z, Whitaker-Menezes D, Howell A, Sotgia F and Lisanti MP: Ketone body utilization drives tumor growth and metastasis. Cell Cycle 11: 3964-3971, 2012.

11. Nieman KM, Kenny HA, Penicka CV, Ladanyi A, BuellGutbrod R, Zillhardt MR, Romero IL, Carey MS, Mills GB, Hotamisligil GS, et al: Adipocytes promote ovarian cancer metastasis and provide energy for rapid tumor growth. Nat Med 17: 1498-1503, 2011.
12. Hancke K, Grubeck D, Hauser N, Kreienberg R and Weiss JM: Adipocyte fatty acid-binding protein as a novel prognostic factor in obese breast cancer patients. Breast Cancer Res Treat 119: 367-377, 2010.

13. Uehara H, Takahashi $\mathrm{T}$, Oha $\mathrm{M}$, Ogawa $\mathrm{H}$ and Izumi $\mathrm{K}$ : Exogenous fatty acid binding protein 4 promotes human prostate cancer cell progression. Int J Cancer 135: 2558-2568, 2014.

14. Kim S, Lee Y and Koo JS: Differential expression of lipid metabolism-related proteins in different breast cancer subtypes. PLoS One 10: e0119473, 2015.

15. Lippman M, Bolan G and Huff K: The effects of glucocorticoids and progesterone on hormone-responsive human breast cancer in long-term tissue culture. Cancer Res 36: 4602-4609, 1976.

16. Goya L, Maiyar AC, Ge Y and Firestone GL: Glucocorticoids induce a G1/G0 cell cycle arrest of Con8 rat mammary tumor cells that is synchronously reversed by steroid withdrawal or addition of transforming growth factor-alpha. Mol Endocrinol 7: 1121-1132, 1993.

17. Rubiś B, Grodecka-Gazdecka S, Lecybył R, Ociepa M, Krozowski Z and Trzeciak WH: Contribution of protein kinase A and protein kinase $\mathrm{C}$ signalling pathways to the regulation of HSD11B2 expression and proliferation of MCF-7 cells. Acta Biochim Pol 51: 919-924, 2004.

18. Song X, Siriwardhana N, Rathore K, Lin D and Wang HC: Grape seed proanthocyanidin suppression of breast cell carcinogenesis induced by chronic exposure to combined 4-(methylnitrosamino)1-(3-pyridyl)-1-butanone and benzo[a]pyrene. Mol Carcinog 49: 450-463, 2010.

19. Koyama K, Myles K, Smith R and Krozowski Z: Expression of the 11beta-hydroxysteroid dehydrogenase type II enzyme in breast tumors and modulation of activity and cell growth in PMC42 cells. J Steroid Biochem Mol Biol 76: 153-159, 2001.

20. Rabbitt EH, Gittoes NJ, Stewart PM and Hewison M: 11beta-hydroxysteroid dehydrogenases, cell proliferation and malignancy. J Steroid Biochem Mol Biol 85: 415-421, 2003.

21. Lipka C, Mankertz J, Fromm M, Lübbert H, Bühler H, Kühn W, Ragosch V and Hundertmark S: Impairment of the antiproliferative effect of glucocorticosteroids by 11beta-hydroxysteroid dehydrogenase type 2 overexpression in MCF-7 breast-cancer cells. Horm Metab Res 36: 437-444, 2004.

22. Smith-Kirwin SM, O'Connor DM, De Johnston J, Lancey ED, Hassink SG and Funanage VL: Leptin expression in human mammary epithelial cells and breast milk. J Clin Endocrinol Metab 83: 1810-1813, 1998

23. O'brien SN, Welter BH and Price TM: Presence of leptin in breast cell lines and breast tumors. Biochem Biophys Res Commun 259: 695-698, 1999.

24. Sheffield L: Malignant transformation of mammary epithelial cells increases expression of leptin and leptin receptor. Endocr Res 33: 111-118, 2008.

25. Ishikawa M, Kitayama J and Nagawa $H$ : Enhanced expression of leptin and leptin receptor (OB-R) in human breast cancer. Clin Cancer Res 10: 4325-4331, 2004.

26. Tessitore L, Vizio B, Jenkins O, De Stefano I, Ritossa C, Argiles JM, Benedetto C and Mussa A: Leptin expression in colorectal and breast cancer patients. Int J Mol Med 5: 421-426, 2000.

27. Cleary MP, Phillips FC, Getzin SC, Jacobson TL, Jacobson MK, Christensen TA, Juneja SC, Grande JP and Maihle NJ: Genetically obese MMTV-TGF-alpha/Lep(ob)Lep(ob) female mice do not develop mammary tumors. Breast Cancer Res Treat 77: 205-215, 2003.

28. Hu X, Juneja SC, Maihle NJ and Cleary MP: Leptin - a growth factor in normal and malignant breast cells and for normal mammary gland development. J Natl Cancer Inst 94: 1704-1711, 2002.

29. Gallagher EJ and LeRoith D: Minireview: IGF, insulin, and cancer. Endocrinology 152: 2546-2551, 2011.

30. Cohen DH and LeRoith D: Obesity, type 2 diabetes, and cancer: The insulin and IGF connection. Endocr Relat Cancer 19: F27-F45, 2012.

31. Tomblin JK and Salisbury TB: Insulin like growth factor 2 regulation of aryl hydrocarbon receptor in MCF-7 breast cancer cells. Biochem Biophys Res Commun 443: 1092-1096, 2014.

32. Salisbury TB, Morris GZ, Tomblin JK, Chaudhry AR, Cook CR and Santanam N: Aryl hydrocarbon receptor ligands inhibit igf-ii and adipokine stimulated breast cancer cell proliferation. ISRN Endocrinol 2013: 104850, 2013. 
33. Dillon RL, Brown ST, Ling C, Shioda T and Muller WJ: An EGR2/CITED1 transcription factor complex and the 14-3-3sigma tumor suppressor are involved in regulating ErbB2 expression in a transgenic-mouse model of human breast cancer. Mol Cell Biol 27: 8648-8657, 2007.

34. Sweeney C, Fambrough D, Huard C, Diamonti AJ, Lander ES, Cantley LC and Carraway KL III: Growth factor-specific signaling pathway stimulation and gene expression mediated by ErbB receptors. J Biol Chem 276: 22685-22698, 2001.

35. Anderson E, Clarke RB and Howell A: Estrogen responsiveness and control of normal human breast proliferation. J Mammary Gland Biol Neoplasia 3: 23-35, 1998.
36. Yang XR, Figueroa JD, Hewitt SM, Falk RT, Pfeiffer RM, Lissowska J, Peplonska B, Brinton LA, Garcia-Closas M and Sherman ME: Estrogen receptor and progesterone receptor expression in normal terminal duct lobular units surrounding invasive breast cancer. Breast Cancer Res Treat 137: 837-847, 2013.

37. McKian KP, Reynolds CA, Visscher DW, Nassar A, Radisky DC, Vierkant RA, Degnim AC, Boughey JC, Ghosh K, Anderson SS, et al: Novel breast tissue feature strongly associated with risk of breast cancer. J Clin Oncol 27: 5893-5898, 2009. 